\title{
Cysteine-390 is the binding site of luminous substance with symplectin, a photoprotein from Okinawan squid, Symplectoteuthis oualaniensis
}

\author{
By Minoru Isobe, ${ }^{* 1, \dagger}$ Masaki Kuse, ${ }^{* 2}$ Naoki TANI, ${ }^{* 3}$ \\ Tatsuya FUJII*3 and Tsukasa MATSUDA ${ }^{* 4}$
}

(Edited by Teruhiko BEPPU, M.J.A.)

\begin{abstract}
Symplectin is a photoprotein from a luminous squid, Symplectoteuthis oualaniensis. It has a luminous substrate, dehydrocoelenterazine (DCZ), linked through a thioether bond with a cysteine residue. We have proven the binding site of luminous substrate in symplectin by using an artificial analogue of DCZ, ortho-fluoro-DCZ (F-DCZ). F-DCZsymplectin emitting strong blue light was reconstituted from apo-symplectin and F-DCZ. Proteolytic digestion of the reconstituted F-DCZ-symplectin afforded peptides including $\mathrm{C}^{390}$ GLK-F-DCZ (amide), which was detected with a house assembled nano-LC-ESI-Q-TOFMS. The chromo-peptide derived from the F-DCZ-symplectin after luminescence showed the lower molecular mass than that before the luminescence by 12 mass units, corresponding to the loss of one carbon atom upon emitting light. Thus, we have concluded that F-DCZ analogue binds to Cys390 in symplectin so as to emit light.
\end{abstract}

Keywords: binding site, fluoro-dehydrocoelenterazine, luminous substrate, nano-LC-MS, photoprotein, symplectin

\section{Introduction}

Symplectin is a photoprotein of luminous squid, Symplectoteuthis oualaniensis, ${ }^{1}$ Tobi-ika in Japanese, which is widely distributed in tropical Pacific Ocean including Okinawan water. Adult squid is about $30-\mathrm{cm}$ in size, and has a yellow photogenic organ on its mantle, which is comprised of a thousand or more small granules. Symplectin is contained in the granules and emits blue light $(470 \mathrm{~nm})$, when stimulated, as shown in Fig. 1.

Symplectin is a $60 \mathrm{kDa}$ protein, and interacts with dehydrocoelenterazine (DCZ: 1) as a luminous substrate in an oxidized form as well as convalently

*1 Institute for Advanced Research, Nagoya University, Aichi, Japan.

*2 Chemical Instrument Division, Research Center for Materials Science, Nagoya University, Aichi, Japan.

$* 3$ Laboratory of Organic Chemistry, Graduate School of Bioagricultural Sciences, Nagoya University, Aichi, Japan.

$* 4$ Laboratory of Molecular Bioregulation, Graduate School of Bioagricultural Sciences, Nagoya University, Aichi, Japan.

$\dagger$ Correspondence should be addressed: M. Isobe, Institute for Advanced Research, Nagoya University, Aichi, Japan (e-mail: isobem@ff.iij4u.or.jp). Accession number of Symplectin is $\mathrm{AB} 447990$. binds a chromophore $\mathbf{2}$ corresponding to the reduced form of $\mathrm{DCZ}{ }^{2}{ }^{2}$ We now postulate that a cysteine residue at the binding site of symplectin attacks to the substrate to form the chromophore (Scheme 1). The chromophore $\mathbf{2}$ is rapidly oxidized at its optimum $\mathrm{pH} 7.8$ in the presence of dioxygen and mono-valent cation $\left(\mathrm{K}^{+}\right.$or $\left.\mathrm{Na}^{+}\right)$to form a short lifetime intermediate $(\mathbf{3}){ }^{3}{ }^{3}$ The intermediate collapses with releasing carbon dioxide to form an amide (4) having electrons in the singlet excited state orbital. Blue light luminescence is emitted while the excited electrons drop to the ground state, and the resulting amide $\mathbf{4}$ is later hydrolyzed to give coelenteramine (5). Apo-symplectin is then regenerated after removal of 4-hydroxyphenylacetic moiety.

It is important to note that normal solventextraction procedures of the photogenic organ homogenates have never provided any chromophore $\mathbf{2}$ or the DCZ analogues to the extract. This fact suggests that the chromophore $\mathbf{2}$ is covalently bound to apo-symplectin. In our previous works, the key issue was the isolation of an acetone adduct of DCZ (6) during the treatment of the homoge- 

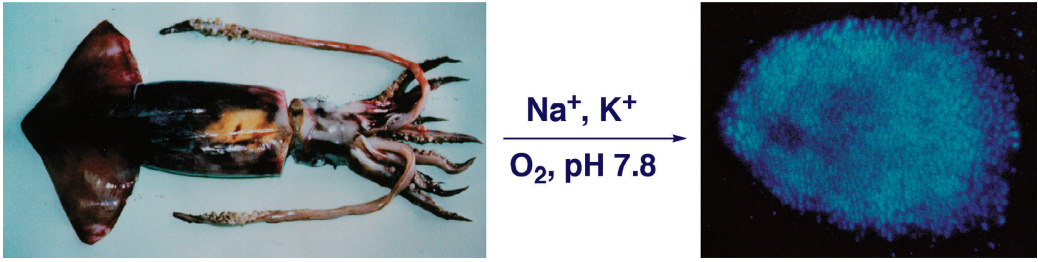

Fig. 1. Luminous squid, Symplectoteuthis oualaniensis, and its blue luminescence.

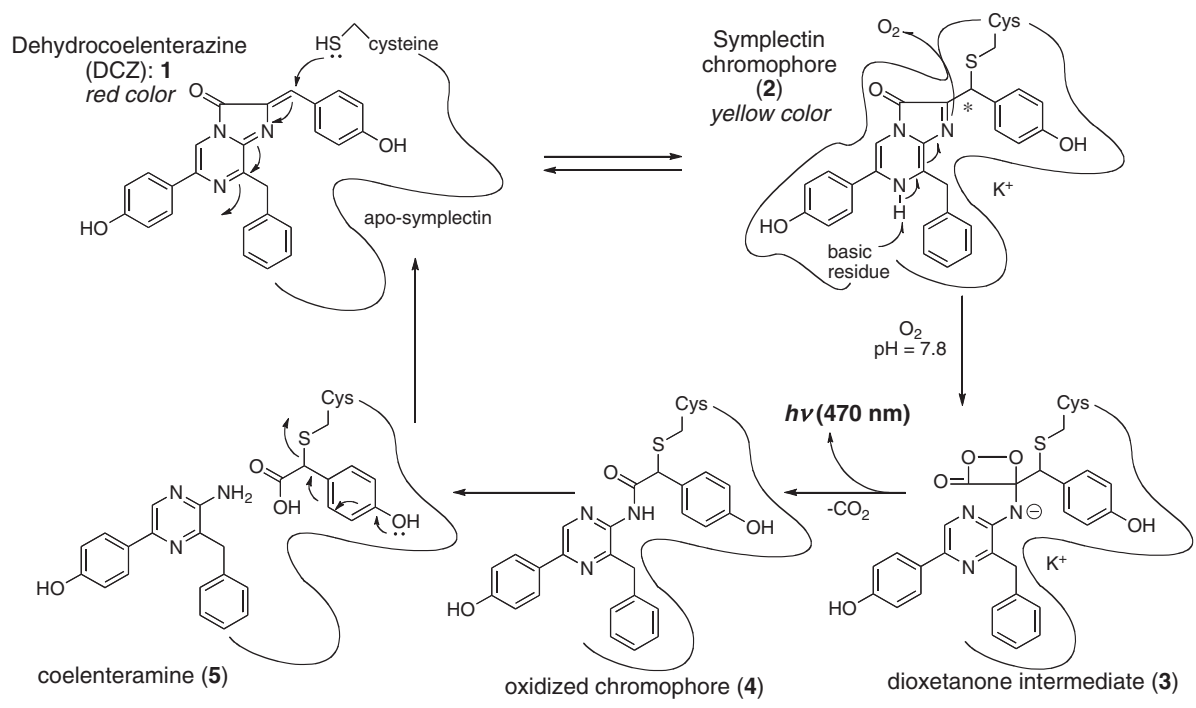

Scheme 1. Proposed molecular mechanism for bioluminescence of symplectin.

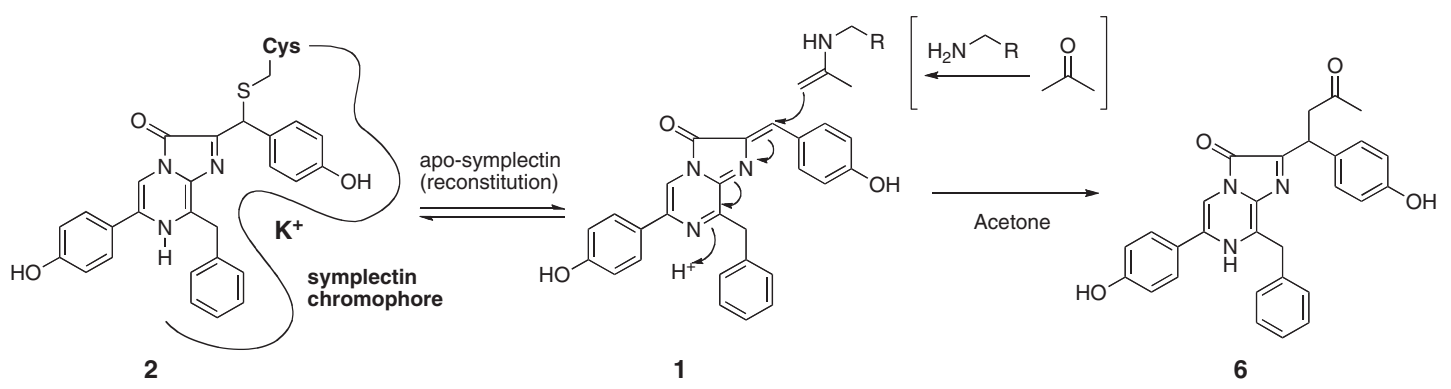

Scheme 2. Isolation of dehydrocoelenterazine (1) from symplectin as the acetone adduct (6).

nates with acetone to make the acetone-powder as shown in Scheme 2. This artifactual compound 6 was only the luminous compound obtained from the $\operatorname{organ}^{2 \mathrm{a})}$

In this paper, we report that Cys-390 links to the luminous substrate as the binding site for bioluminescence.

\section{Results and discussion}

First, we homogenized photogenic organ with
$0.4 \mathrm{M} \mathrm{KCl}$ (in $50 \mathrm{mM}$ phosphate buffer at $\mathrm{pH} 6.0$ containing EDTA and DTT) at $5{ }^{\circ} \mathrm{C}$. After centrifugation, the non-luminescent extract was removed as the supernatant. The remaining precipitates were repeatedly washed with $0.4 \mathrm{M} \mathrm{KCl}$ solution two more times, and then the residue was extracted with $0.6 \mathrm{M} \mathrm{KCl}$ solution. Centrifugation of this extract afforded a symplectin in the supernatant (Scheme 3A). By adjusting $\mathrm{pH}$ from 6.0 to the optimum $\mathrm{pH} 7.8$ by addition of $\mathrm{pH} 8.56$ buffer, 


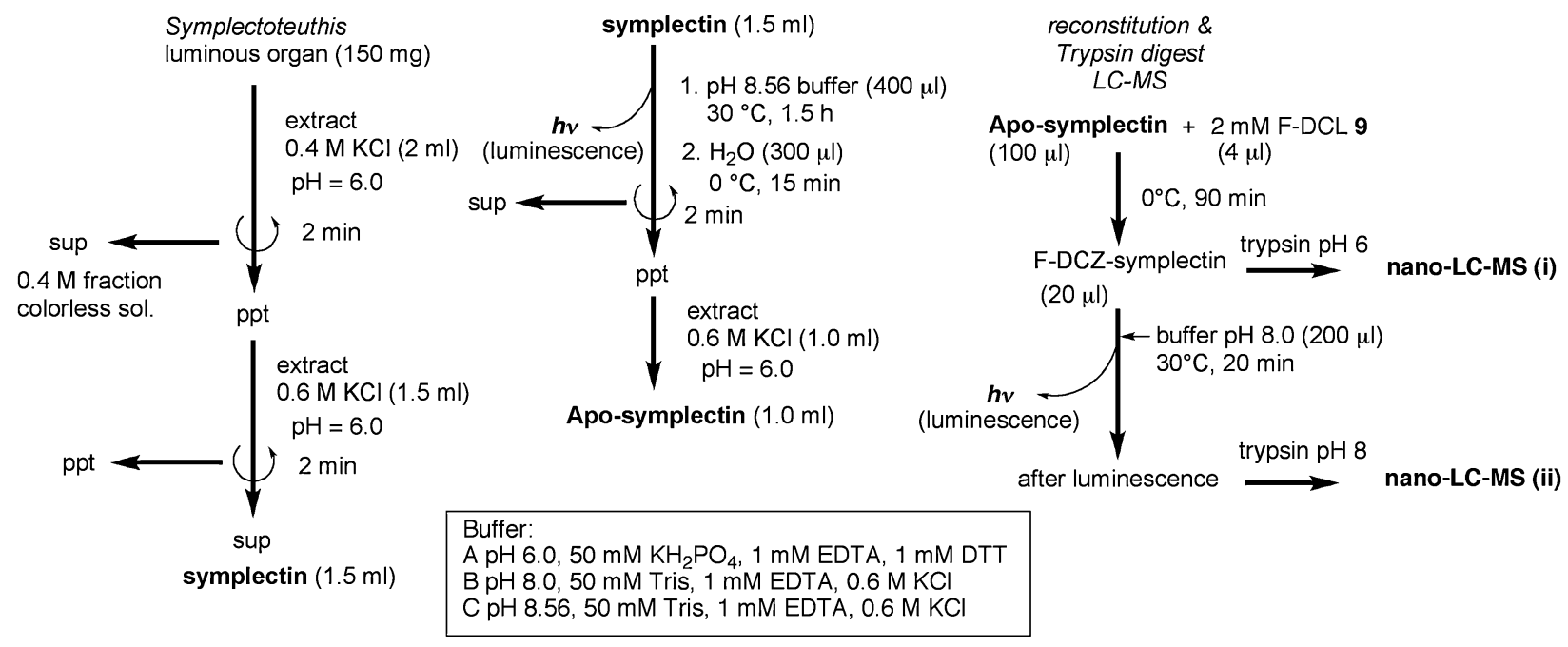

Scheme 3. Procedures for extraction of symplectin from photogenic organ (A), for preparation of apo-symplectin (B), and for reconstitution of F-DCZ-symplectin before and after luminescence (C).

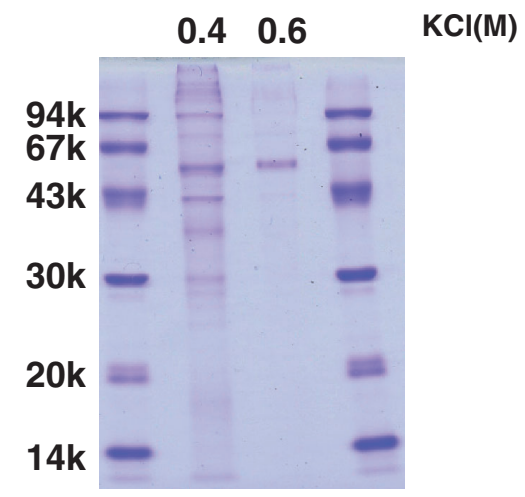

Fig. 2. SDS-PAGE of the extracts with buffer containing $0.4 \mathrm{M}$ and $0.6 \mathrm{M} \mathrm{KCl}$. The band of symplectin appears in the $0.6 \mathrm{M}$ $\mathrm{KCl}$ extract at $60 \mathrm{kDa}$.

strong bioluminescence $(h \nu)$ was observed.

As shown in Fig. 2, SDS-PAGE analysis of symplectin gave almost a single band of $60 \mathrm{kDa}$ without any further purification. After a western blotting to a PVDF membrane, the $60 \mathrm{kDa}$ band showed fluorescence when irradiated with a UV light $(360 \mathrm{~nm})$, indicating the presence of the chromophore or the bound luminescent substrate (Fig. 3).

Partial hydrolysis of symplectin with trypsin afforded two big fragments, whose sizes were $40 \mathrm{kDa}$ and $15 \mathrm{kDa}$, respectively. ${ }^{4)}$ The $40 \mathrm{kDa}$ fragment still had bioluminescent activity as well as fluores- cence, suggesting that the presence of the bound luminous substance. We then analyzed the amino acid sequence of symplectin with Edman degradation, nano-LC-ESI-Q-TOF-MS and MS/MS analysis, ${ }^{5)}$ as well as cloning and sequencing of the cDNA. Figure 4 shows the total amino acid sequence of symplectin. ${ }^{6}$ )

As proved by these analyses, symplectin consists of 501 amino acids and total of 11 cysteine residues in the sequence as shown in Fig. 4. The Nterminal sequence of the $40 \mathrm{kDa}$ trypsin fragment

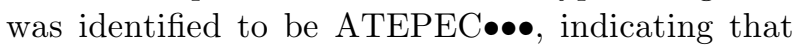
the fragment corresponds to the C-terminal part of symplectin, while the $15 \mathrm{kDa}$ fragment to the $\mathrm{N}$ terminal part. Breakage by trypsin digestion occurred at the Arg-131. The $40 \mathrm{kDa}$ fragment has 8 cysteine residues, one of which must bind DCZ to form the chromophore in a reduced form for bioluminescence. We further found that digestion of symplectin with V8 protease gave another fluorescent peptide. Nano-LC-ESI-MS/MS analysis of the peptide revealed that the $\mathrm{N}$-terminal sequence was identical with the sequence from Val-358,

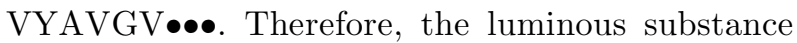
should be bound to any one of Cys-380, Cys-385, or Cys-390.

During the course of determining the binding site cysteine, we found that the bound chromophore $\mathbf{2}$ in symplectin is under the reversible exchange 


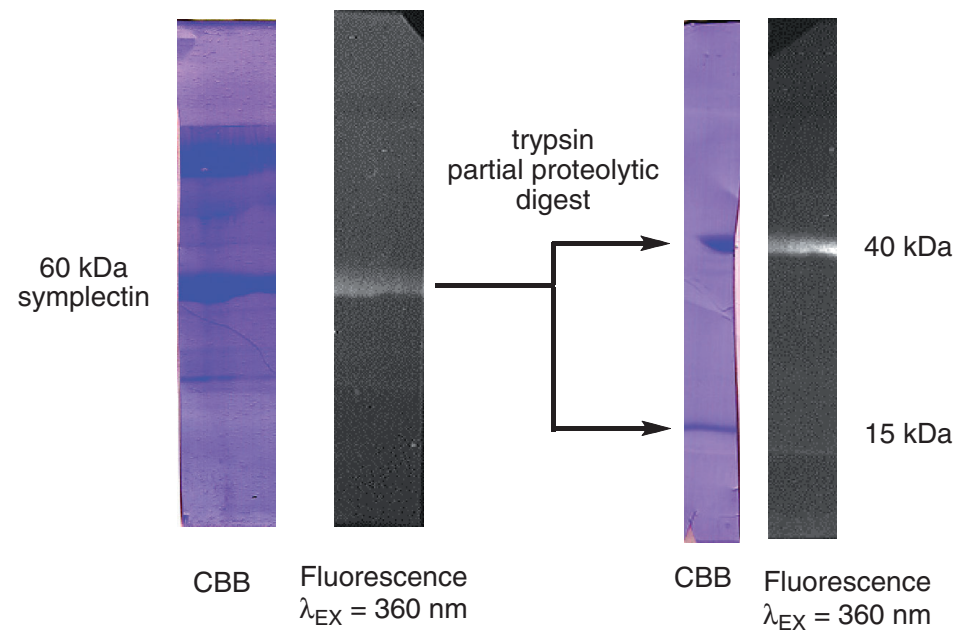

Fig. 3. SDS-PAGE showing cleavage of symplectin $(60 \mathrm{kDa})$ to the $40 \mathrm{kDa}$ and $15 \mathrm{kDa}$ fragments by partial tryptic digestion. The protein bands were blotted to PVDF membranes and illuminated with UV at $360 \mathrm{~nm}$.

\begin{tabular}{|c|c|c|c|c|}
\hline & 11 & 21 & 31 & 41 \\
\hline TRPVSSWKV & AVFEHQVIPP & KTDMETREEA & LDALKLNSDV & YHE \\
\hline GVKMIVFPE & YGLYDINTLT & RTRMDLMAEK & VPHPKHGHRN & PCDEF \\
\hline SEI & AKENDMYMV & VNMAGREPCR & RATEPECPGD & $\mathrm{KQI}$ \\
\hline NNEGDVVAR & YYKTHLFWEE & GWFNSSKNYE & MALWDTPIGK & FGTF \\
\hline YNV & RHIAYPASWV & NLPPIYQSIQ & SHSAFARFAK & INLI \\
\hline UETSTYGSGI & YSPNGAEIFY & FRPDIPKSKL & LVAEILPIHV & KKPEQTVVNF \\
\hline NPVFPSEDD & DVQDLFDRGD & FAFLKYKRMT & TRAGTVEVCQ & KSFCCKARY \\
\hline TKDRFKEVYA & VGVYDGLLSA & GANNLYFQIC & TVIQCPHKKC & GLKISKVRT \\
\hline FYLNLRADG & WLDRYVFPSY & TVMYNNYIAL & DPFVWNYTVA & GGIETKPGT \\
\hline $\begin{array}{l}\text { TPLHSANLVA } \\
\mathrm{S}\end{array}$ & RIYAKDSSKH & VHQPHPIDEG & VIKMAVKYML & YVMAAYVYA \\
\hline & & & & \\
\hline
\end{tabular}

Fig. 4. Amino acid sequence of symplectin. The sequences of the $15 \mathrm{kDa}$ and $40 \mathrm{kDa}$ fragments are indicated in blue and black letters, respectively. All cysteine residues are in red. A large squared area with a thin red line indicates the fragment by V8 protease. The peptide containing Cys-390 to bind DCZ is indicated with an arrow.
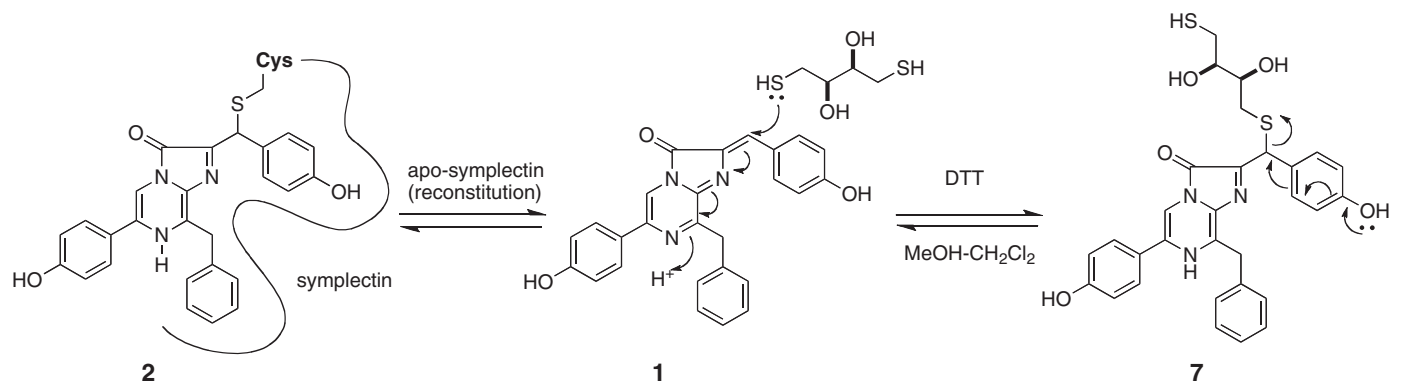

Scheme 4. Equilibrium between free DCZ and thiol adducts with apo-symplectin or with DTT.

with free DCZ (1) and the presence of DTT enhances releasing the chromophore $\mathbf{2}$ as the thioladduct $\mathbf{7}$ to give apo-symplectin according to the Scheme 4. This equilibrium made it difficult to determine the binding site in symplectin, because the chromophore might be released from the peptides during the proteolytic digestion. To overcome this dissociation problem, we employed an 


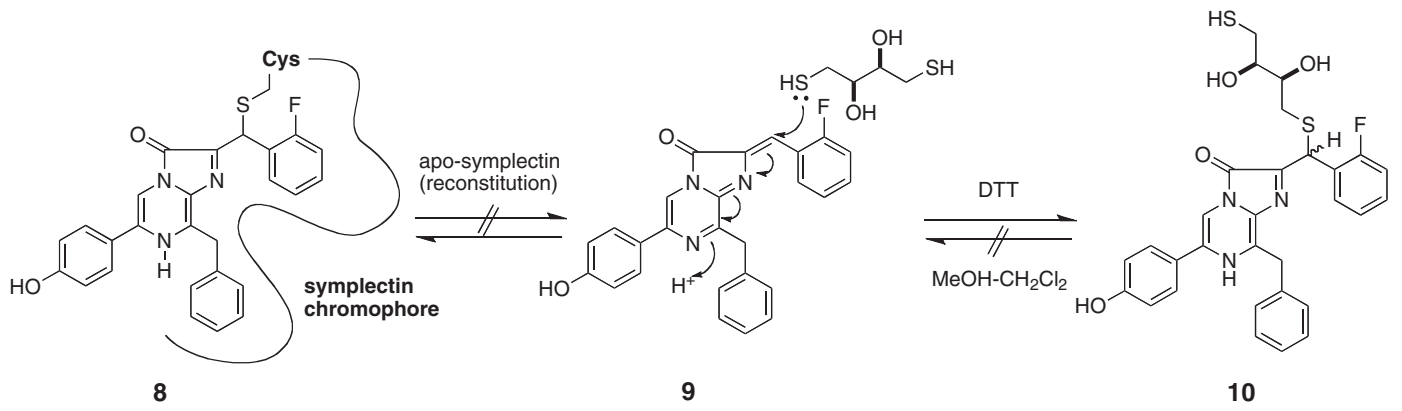

Scheme 5. Stable adduct formation of F-DCZ with apo-symplectin or with DTT.

artificial DCZ, a fluorinated analogue F-DCZ, 9, which makes more stable binding (8) with a cysteine residue in the peptides due to the electron withdrawing characteristics of fluorine. Among the three mono-fluoro analogues of DCZ (F atom located at ortho, meta, or para position), ${ }^{7)}$ we selected the ortho-fluoro-DCZ (9) due to its strongest bioluminescence in the reconstituted protein. We then confirmed that the equilibrium between free F-DCZ (9) and its thiol-adduct (8 or 10) with free DTT or apo-symplectin extremely inclined toward the adduct formation. In fact, we succeeded in isolating its stable adduct with DTT (10) by HPLC and identified its two diastereoisomers by using nano-LC-Q-TOF MS and MS/MS.7),8) These experiments suggest that the analogue bound to the cysteine residue in apo-symplectin also remains stable and chromophore-binding peptide may be analyzed by using this non-natural analogue.

Considering the structural similarity of the both natural (1) and non-natural dehydrocoelenterazine $(\mathbf{9})$, we may reasonably assume that the same site in symplectin is involved for their binding. Then we reconstituted F-DCZ-symplectin from apo-symplectin and $\mathbf{9}$ at $\mathrm{pH} 6.0$ according to the process shown in Scheme 3B, which corresponds to the molecular form before luminescence (2) in Scheme 1. The reconstituted protein was subjected to the extensive tryptic digestion and the resultant peptides were analyzed with nano-HPLC-ESI-MS. Among 51 peaks of the peptides (T1-T52) thus obtained, only one peak of T43 eluted at $19.23 \mathrm{~min}$ on nano-HPLC afforded a doubly charged molecular ion at $422.28(\mathrm{M}+2 \mathrm{H})^{++}(\mathrm{MW} 843.55)$, which was assignable to the chromo-peptide $\mathrm{C}^{390} \mathrm{GLK}$ forming the stable adduct with F-DCZ (11) (Fig. 5).

On the other hand, the reconstituted F-DCZ- symplectin was incubated in the presence of $50 \mathrm{mM}$ $\mathrm{K}^{+}$for $20 \mathrm{~min}$ at $\mathrm{pH} 8.0$ so as to emit strong blue light (Scheme $3 \mathrm{C}$ ), and the protein corresponding to the form after luminescence (4) was recovered. In the similar analysis of its tryptic digest, a peptide eluted at $20.96 \mathrm{~min}$ was found to afford a doubly charged ion at $416.27(\mathrm{M}+2 \mathrm{H})^{++}(\mathrm{MW} 831.53)$, which was assigned to the same peptide $\mathrm{C}^{390} \mathrm{GLK}$ forming adduct with oxidized F-DCZ (12). The result indicates loss of 12 mass units or 1 carbon atom from the chromo-peptide during luminescence, which coincides with the expected structural change (from $\mathbf{2}$ to 4 ) through the oxygenated intermediate (3) (Scheme 1).

According to these nano-LC mass spectrometric analyses with fluorinated chromo-peptides before and after the luminescence, we conclude that Cys-390 among 11 cysteine residues in symplectin is the binding site for F-DCZ $(\mathbf{9})$ through a thioether (-S-) bond to form an adduct with structure 8. A nano-LC-ESI-Q-TOF-MS system, which we had developed for trace analyses, ${ }^{5), 8)}$ played an essential role to conduct these works.

Experimental instrumentation: ${ }^{9)}$ The nanoLC-MS were performed on a Q-TOF mass spectrometer (Micromass, Manchester, UK) fitted with a Z-spray-type ESI (electrospray ionization) source, with resolution ca. 8,500 mass unit. Data were acquired in the positive ion mode, and processed with MassLynx version 3.4. LC-MS was conducted with appropriately modified nano-HPLC system (JASCO, Tokyo, Japan). A separation column, Develosil ODS-HG-5 (Nomura Chem., Seto, Aichi, Japan) of a size $0.3 \mathrm{~mm}$ i.d. $\times 15 \mathrm{~cm}$, was used with a linear gradient PPG (prepacked gradient in a capillary tubing of 50 micrometer i.d. $\times 500 \mathrm{~cm}$ ) elution system from $0 \%$ to $100 \%$ acetonitrile/water 


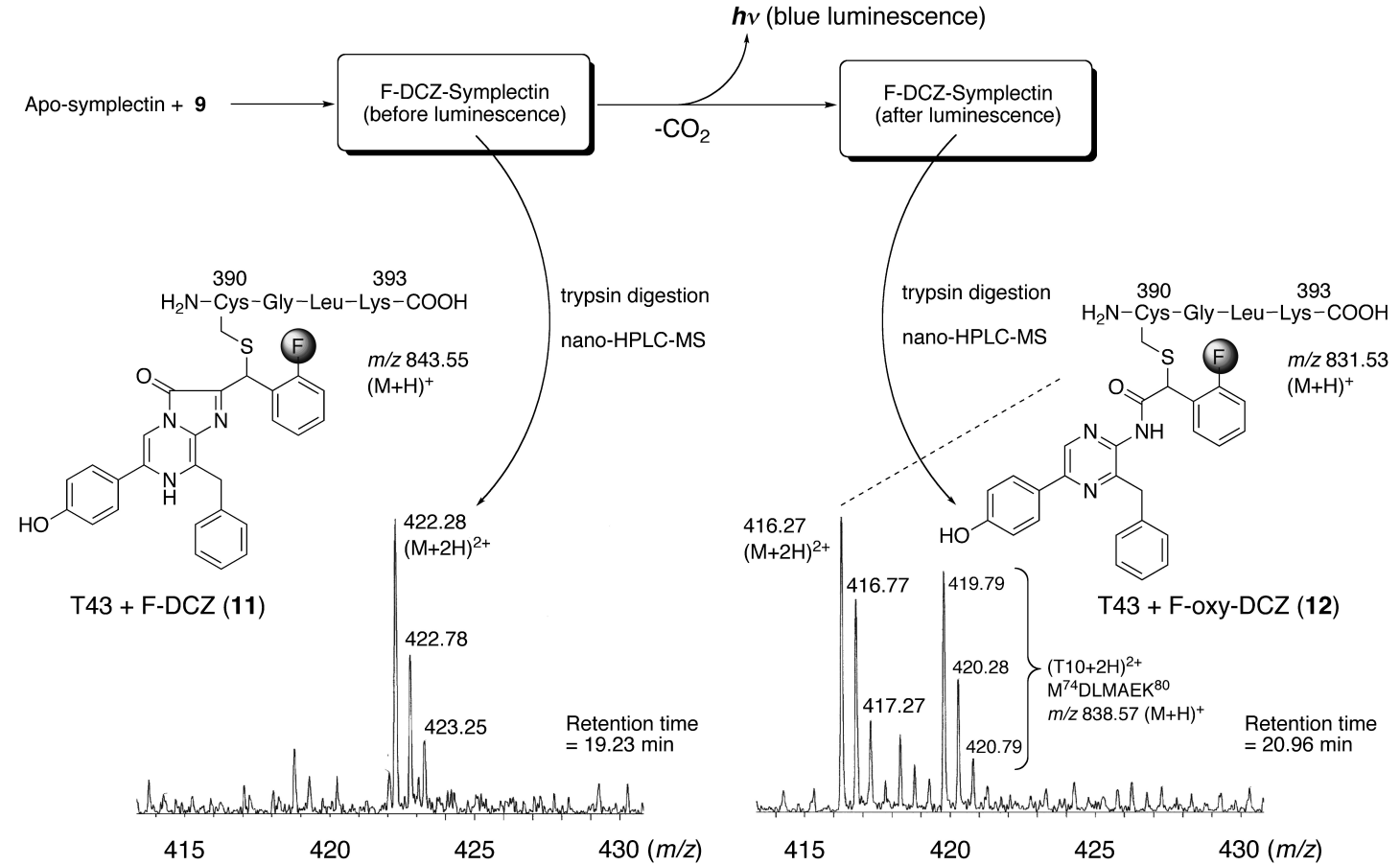

Fig. 5. Nano-LC-ESI-Q-TOF-MS spectra of the chromo-peptide in the tryptic digests of F-DCZ-symplectin before and after the luminescence. Chromo-peptide before luminescence $(\mathbf{1 1}, \mathrm{m} / z$ 843.55) shifted to $\mathbf{1 2}(\mathrm{m} / z$ 831.53) after luminescence. Molecular ions at $m / z 419.79$ corresponding to peptide $\mathrm{T} 10=\mathrm{M}^{74}$ DLMAEK $^{80}$ was observed at the same retention time with 12 (Those masses were observed as doubly charged ions.).

containing trifluoroacetic acid $(0.025 \%)$ for $40 \mathrm{~min}$ at a flow rate of $5 \mu \mathrm{l} / \mathrm{min}$ without flow splitting. The column effluent was monitored at $210 \mathrm{~nm}$ with a capillary UV detector and was then introduced into the electrospray nebulizer.

Preparation of symplectin sample: ${ }^{10)}$

Whole photogenic organ was removed from 10-20 luminous squid, Symplectoteuthis oualaniensis, and lyophilized and kept at $-80{ }^{\circ} \mathrm{C}$. A $150 \mathrm{mg}$ of dry organ was homogenized in acetone at $-78^{\circ} \mathrm{C}$ and then suspended in a cold buffer A $(2 \mathrm{ml} \mathrm{pH} 6.0$, $50 \mathrm{mM} \mathrm{KH_{2 }} \mathrm{PO}_{4}, 1 \mathrm{mM}$ EDTA, $1 \mathrm{mM}$ DTT) containing $0.4 \mathrm{M} \mathrm{KCl}$ and centrifuged at $10,000 \mathrm{~g}$ for $10 \mathrm{~min}$. The precipitate was repeatedly washed one more time as above. The precipitate was then resuspended in buffer A containing $0.6 \mathrm{M} \mathrm{KCl}$, homogenized, and centrifuged again. The resultant supernatant $(1.5 \mathrm{ml})$ was used as symplectin solution. To this solution was added buffer C $(400 \mu \mathrm{l}$, $\mathrm{pH} 8.56,50 \mathrm{mM}$ Tris, $1 \mathrm{mM}$ EDTA) containing $0.6 \mathrm{M} \mathrm{KCl}$, and kept at $30^{\circ} \mathrm{C}$, while strong luminescence was observed in the dark. After $1.5 \mathrm{hr}$, the solution was diluted by addition of water $(0.3 \mathrm{ml})$ and kept in ice bath for 15 min to find precipitates. The precipitates were collected by centrifugation, and extracted by $0.6 \mathrm{M} \mathrm{KCl}$ buffer A (1 ml). This solution was termed Apo-symplectin.

To the apo-symplectin $(100 \mu \mathrm{l})$ was added $4 \mu \mathrm{l}$ of DMSO solution of F-DCL $(9,2 \mathrm{mM})$, and kept at $0{ }^{\circ} \mathrm{C}$ for $1.5 \mathrm{hr}$ to obtain F-DCZ-symplectin. A part of this solution $(20 \mu \mathrm{l})$ was subjected to digestion by addition of $2 \mu \mathrm{g}$ trypsin $(200 \mu \mathrm{l} \mathrm{pH} 6.0$, buffer A) for $12 \mathrm{hr}$ at $30^{\circ} \mathrm{C}$ with shaking. The resulting tryptic digests were analyzed by a nano-HPLC-MS instrument by injecting ca. $2 \mu$ lo a pre-column (ODSUG, $0.5 \mathrm{~mm}$ i.d. $\times 3 \mathrm{~cm}$ for desalting) and reversely eluted from the pre-column by gradient solvent in the packed capillary through a capillary-valve into a LC column (ODS-UG, $0.3 \mathrm{~mm}$ i.d. $\times 15 \mathrm{~cm}$ ), then into capillary UV detector and to the ESI-Q-TOF mass spectrometer. The peptides were detected in the amount ranging from 0.01 to 10 picomole levels.

Another F-DCL-symplectin $(20 \mu \mathrm{l})$ was diluted

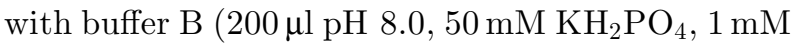
EDTA, $0.6 \mathrm{M} \mathrm{KCl}$ ) and kept for $20 \mathrm{~min}$ at $30{ }^{\circ} \mathrm{C}$ for luminescence. The resultant solution was then 
digested with $2 \mu \mathrm{g}$ trypsin $(20 \mu \mathrm{l}$ buffer B) for $12 \mathrm{hr}$ under the same condition as above and similarly analyzed by a nano-HPLC-ESI-Q-TOF-MS and the results are shown in Fig. 5.

\section{Acknowledgements}

The authors acknowledge the financial supports, a Grant-in-Aid for Specially Promoted Research [16002007 (2004-8)] from the Ministry of Education, Culture, Sports, Science and Technology (MEXT), Japan. We also thank for the financial support to MK from a Grant-in-Aid for Young Scientists (B) [19780087 (2007)] from MEXT.

\section{References}

1) Tsuji, F.I. and Leisman, G. (1981) $\mathrm{K}^{+} / \mathrm{Na}^{+}$-triggered bioluminescence in the oceanic squid Symplectoteuthis oualaniensis. Proc. Natl. Acad. Sci. USA 78, 6719-6723.

2) a) Takahashi, H. and Isobe, M. (1993) Symplectoteuthis Bioluminescence (1). Structure and binding form of chromophore in photoprotein of a luminous squid. Bioorg. Med. Chem. Lett. 3, 2647-2652; b) Takahashi, H. and Isobe, M. (1994) Photoprotein of luminous squid, Symplectoteuthis oualaniensis and reconstruction of the luminous system. Chem. Lett. 23, 843-846; c) Isobe, M., Fujii, T., Swan, S., Kuse, M., Tsuboi, K., Miyazaki, A., Feng, M.C. and Li, J. (1998) Chemistry of photoproteins as interface between bioactive molecules and protein function. Pure \& Appl. Chem. 70, 2085-2092; d) Isobe, M., Kuse, M., Yasuda, Y. and Takahashi, H. (1998) Synthesis of ${ }^{13} \mathrm{C}$-dehydrocoelenterazine and model studies on Symplectoteuthis squid bioluminescence. Bioorg. Med. Chem. Lett. 8, 2919-2924; e) Kuse, M. and Isobe, M. (2000) Synthesis of ${ }^{13} \mathrm{C}$-dehydrocoelenterazine and NMR studies on the bioluminescence of a Symplectoteuthis model. Tetrahedron 56, 2629-2639.

3) a) Usami, K. and Isobe, M. (1995) Two luminescent intermediates of coelenterazine analog, peroxide and dioxetanone, prepared by direct photo-oxy- genation at low temperature. Tetrahedron Lett. 36, 8613-8616; b) Usami, K. and Isobe, M. (1996) Low-temperature photooxygenation of coelenterate luciferin analog synthesis and proof of 1,2dioxetanone as luminescence intermediate. Tetrahedron 52, 12061-12090.

4) Fujii, T., Ahn, J.Y., Kuse, M., Mori, H., Matsuda, T. and Isobe, M. (2002) A novel $60 \mathrm{kDa}$-photoprotein from oceanic sqiud (Symplectoteuthis oualaniensis) with sequence similarity to mammalian carbon-nitrogen hydrolase domains. Biochem. Biophys. Res. Commun. 293, 874-879.

5) Kurahashi, T., Miyazaki, A., Suwan, S. and Isobe, M. (2001) Extensive investigations on oxidized amino acid residues in $\mathrm{H}_{2} \mathrm{O}_{2}$-treated $\mathrm{Cu}$.Zn-SOD protein with LC-ESI-Q-TOF-MS, MS/MS for the determination of the copper-binding site. J. Am. Chem. Soc. 123, 9268-9278.

6) Isobe, M. and Matsuda, T. (2000) JP Patent 2000154786. Accession number of symplectin is AB447990. The S-S bondings are located between Cysteines 92-110, 129-137, and 380-385, respectively. The details of these informations are to be reported elsewhere in due course.

7) Isobe, M., Fujii, T., Kuse, M., Miyamoto, K. and Koga, K. (2002) ${ }^{19} \mathrm{~F}$-Dehydrocoelenterazine as probe to investigate the active site of symplectin. Tetrahedron 58, 2117-2126.

8) Sydnes, M.O., Kuse, M., Kurono, M., Shimomura, A., Ohinata, H., Takai, A. and Isobe, M. (2008) Protein phosphatase inhibitory activity of tautomycin photoaffinity probes evaluated at femtomolar level. Bioorg. Med. Chem. 16, 1747-1755.

9) These conditions are essentially identical as reported in experimental part in Ref. 5 and following paper; Isobe, M., Kai, H., Kurahashi, T., Suwan, S., Pitchayawasin-T. S., Franz, T., Tani, N., Higashi, K. and Nishida, H. (2006) The molecular mechanism of the termination of insect diapause, Part 1: A timer protein, TIME-EA4, in the diapause eggs of the silkworm Bombyx mori is a Metallo-Glycoprotein. ChemBioChem. 7, 15901598.

10) These conditions are essentially identical as reported in Refs. 4 and 7.

(Received July 16, 2008; accepted Oct. 7, 2008) 